\title{
Offensive Positioning Based on Maximum Weighted Bipartite Matching and Voronoi Diagram
}

\author{
Mohammadhossein Malmir $\left.{ }^{(}\right)$, Shahin Boluki, and Saeed Shiry Ghidary \\ Amirkabir University of Technology (Tehran Polytechnic), Hafez Ave., Tehran, Iran \\ \{mhmalmir, sh.boluki, shiry\}@aut.ac.ir
}

\begin{abstract}
In this paper we propose a modification to the well known Delaunay Triangulation based positioning in the attacking situation positioning of the agents in 2D Soccer Simulation environment. Due to advanced defensive skills such as marking skill, the attacker agents should have a dynamic positioning with respect to the rival team defenders. The proposed method employs the vertices of the Voronoi Diagram of the defending team agents as potential positions for the attacker team agents, since these positions are dynamic and change with the movement of the defending team agents and are always safe positions regarding the distance to the defending team agents, and also have a good coverage of the field. So the attacking agents can increase the chance of receiving pass by the ball owner agent and the scoring chance by taking positions on these vertices. This proposed method then applies Maximum Weighted Bipartite Matching to match these vertices to the attacking agents. This algorithm can be applied by each agent individually, but in order to reduce the possible decision conflicts in this matching which is the result of the limitation in the incoming information of the field from the agents' sensors, this algorithm can be performed by one agent and then this agent should inform the other attacking team agents of the result by communication skills like "say ability" in 2D Soccer Simulation (SS) environment. This method shows better performance in offensive situation than the conventional Delaunay Triangulation based positioning. It is tested in 2D SS environment as a highly dynamic multi-agent environment but its application is not restricted to the 2D SS League.
\end{abstract}

Keywords: Delaunay Triangulation - Voronoi diagram - Maximum weighted bipartite matching $\cdot$ Offensive positioning

\section{Introduction}

Positioning involves finding the best target position for agents who do not possess the ball regarding the field situation and team strategy [1]. Positioning is of great importance in a soccer match, either in defensive situation or in offensive situation. Soccer league of Robocup is no exception. In offensive positioning which is the case when one agent of us owns the ball, all the other agents of us 
should modify their position in field in order to increase the chance of receiving pass from the ball owner and increase the scoring chance. We propose and test our algorithm for offensive positioning on 2D Soccer Simulation environment due to the fact that in 2D Soccer Simulation we have a multi-agent system that our agents do not have the limitations of real robots in their movement, nevertheless the maximum acceleration and speed of movement of agents, and the similar energy factor of humans are simulated by the stamina factor and other parameters in this simulated environment. Therefore, considering the stamina and smooth movement in positioning is a crucial task. The problem of positioning within the Robocup context has been widely investigated in the past years and the methods vary a lot and it seems that there is no standard approach [2]. Some differences are surely influenced by the special specifications of each league of Robocup. A survey of approaches is out of the scope of this paper, but a nearly complete list of important approaches can be found in $[1,2]$. In general the solutions within the simulation leagues are more elaborated and computationally complex in comparison to solutions within hardware leagues due to the more reliable world model and less motion and low level control considerations within the simulation leagues [2]. In this paper we focus our attention to a widely used algorithm of positioning in Robocup Soccer Simulation League, Delaunay Triangulation based positioning depending on the ball's position in the field, and we propose a modification to improve the offensive situation positioning of agents. In 2008 Hidehisa Akiyama and Itsuki Noda used Delaunay Triangulation of the field depending on the ball position to determine the agents' positions [3-5]. In this method a representative set of potential ball positions are the vertices of these triangles and in each potential ball position we can set the positions of all of our agents. During the match the positions of the agents are determined by an interpolation method between the vertices of the triangle in which the current position of the ball lies. This method has shown a great performance and was adopted by most of the teams participating in Soccer Simulation League of Robocup Competitions in recent years. One of the benefits of this method is its simplicity in implementation and initialization by the developer, since it can be done by a visual software namely fedit provided by Hidehisa Akiyama [6] and all the considerations of smooth movement of players between positions can be handled by the human intuition operation in a visual manner. In Fig. 1 the Delaunay Triangulation of the field based on the representative set of ball positions in the GUI of fedit is shown. In each numbered potential position of the ball, the human developer determines the positions of all the agents. But this method has a major defect that it does not consider the opponent agents and is static with respect to opponent players. Due to recent defensive skill advancements and strong marking skill of Soccer Simulation Teams, receiving passes in the opponent's penalty area and making scoring chances requires a dynamic positioning regarding the opponent's agents to escape their marking skills. In this paper we propose an algorithm to achieve this goal in offensive positioning by applying Voronoi Diagram of opponent's agents and Maximum Weighted Bipartite Matching [7-10]. To be more precise, the proposed method 


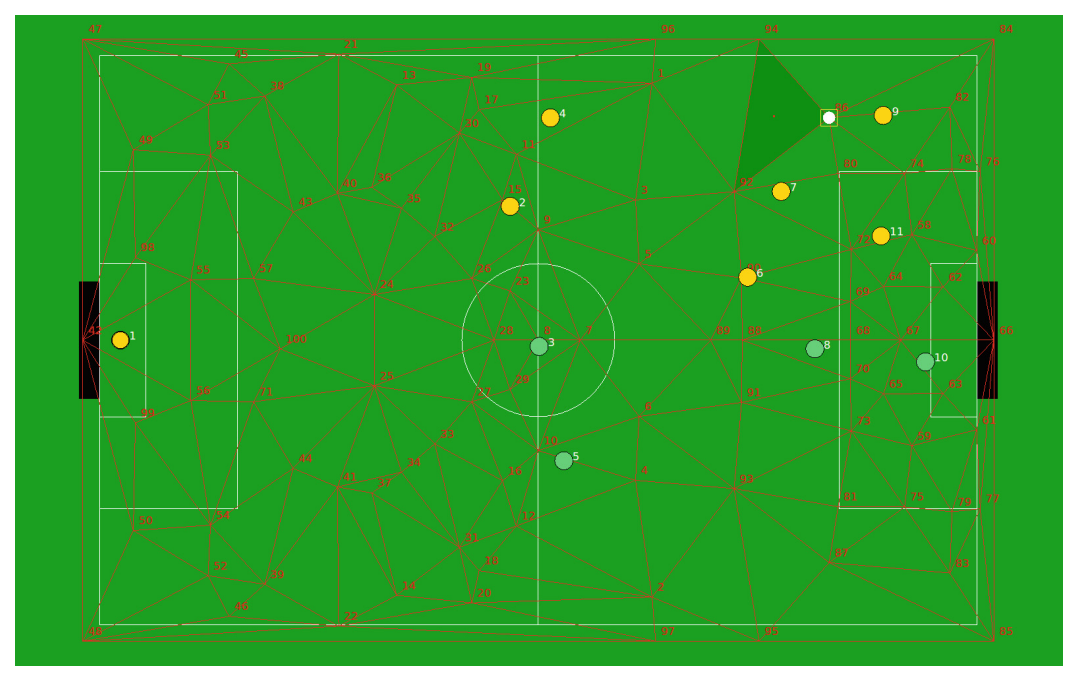

Fig. 1. Agents' positioning based on Delaunay triangulation.

considers the vertices of the defending team agents' Voronoi Diagram as potential positioning points for attacking team agents, since they have a good and safe distance from all the defending players and positioning in these points increase the chance of receiving passes by teammate agents. This algorithm then makes a bipartite graph, assuming these points on one side and attacking team agents on the other side, so our problem reduces to an assignment problem to determine which agent should choose which vertex to take position on. Besides, Soccer Simulation environment is a multi-agent system in which we have an increased chance of decision conflicts when each agent applies the algorithm and reaches to a conclusion individually, due to the limitations and uncertainties in each agent's information of the field situation gathered by its sensors. For this reason in the implementation of this algorithm one agent which has the best and most confident information of the field applies the algorithm and informs the other teammate agents of the result by the "say ability". In the proceeding we first give a short description of Maximum Weighted Bipartite Matching and Voronoi Diagram, and then we describe our algorithm in detail. In Sect. 5 we define and employ performance measures to compare our algorithm with the conventional Delaunay Triangulation employed currently by most of the soccer simulation teams regarding offensive positioning in opponent's half-field and near goal situations. At the end a conclusion is presented in Sect. 6.

\section{Maximum Weighted Bipartite Matching}

Graph $G=(V, E)$ in which $V$ is the set of vertices and $E$ is the set of edges is called Bipartite if the set $V$ can be divided into two parts $A$ and $B$ such that,

$$
A \cap B=\emptyset
$$




$$
A \cup B=V,
$$

and also there does not exist any edge in $E$ that connects two vertices in the same set [7]. A subset $M$ of the set $E$ is a matching (collection of edges) when each vertex of $V$ is at most incident to one edge of $M$. Without loss of generality we can assume our graph complete by adding dummy vertices and edges of weight zero $[7,8]$.

If each edge of the graph is assigned a weight we have a weighted bipartite graph [11]. If the sum of the weights of the edges in a matching $\left(M_{i}\right)$ is called the weight of that match $W\left(M_{i}\right)$,

$$
W\left(M_{i}\right)=\sum_{e \in M_{i}} w(e),
$$

a maximum weighted matching $M$ is a match in such a way that every other matching has lower weight than the weight of $M$ [12]. In the proposed algorithm we employ Hungarian method to solve our assignment problem. This Maximum Weighted Bipartite Matching has been used in defensive skill in $[11,12]$ as well.

\section{Voronoi Diagram}

The Voronoi diagram is a versatile geometric structure and has many applications in social geography, physics, astronomy, robotics, and many more fields $[13,14]$. The following description and the rest of this section is a summary of the definition originally presented in [15].

Let $S$ denote a set of $n$ sites (e.g., defending team's agent positions) in the two-dimensional plane. For two distinct sites $p, q \in S$, the dominance of $p$ over $q$ is defined as the subset of the plane being at least as close to $p$ as to $q$, that is,

$$
\operatorname{dom}(p, q)=\left\{x \in \mathbb{R}^{2} \mid d(x, p) \leq d(x, q)\right\}
$$

where $d(x, p)$ is the Euclidean distance between two points $x$ and $p$. Let $\overline{\mathrm{pq}}$ denote the line segment between $p$ and $q$. The perpendicular bisector of $\overline{\mathrm{pq}}$ divides the plane into two halves. This perpendicular bisector, denoted by $\overline{\mathrm{pq}}{ }^{\perp}$, is called the separator of $p$ and $q$. We denote the open half-plane that contains $p$ by $h_{p}(q)$. Any point on the separator $\overline{\mathrm{pq}}^{\perp}$ is equidistant to $p$ and $q$. Any point within a half plane $h_{p}(q)$ has the distance to $p$ smaller than the distance to $q$. Therefore, $\operatorname{dom}(p, q)$ is the perpendicular bisector line $\overline{\mathrm{pq}}^{\perp}$ plus the half of the plane $h_{p}(q)$. The region of a site $p \in S$ is the portion of the plane lying in all the dominance of $p$ over the remaining sites in $S$, that is,

$$
\operatorname{reg}(p)=\cap_{q \in S-\{p\}} \operatorname{dom}(p, q)
$$

The region $\operatorname{reg}(p)$ comes from intersecting $n-1$ half planes, and it is a convex polygon. Furthermore, the boundary of $\operatorname{reg}(p)$ consists of at most $n-1$ edges 
(maximal open straight-line segments) and vertices (their endpoints). Each point on an edge is equidistant from exactly two sites, and each vertex is equidistant from at least three sites. As a consequence, the edges and vertices of all regions form a polygonal division of the whole plane. This partition is called the Voronoi diagram, $\operatorname{Vor}(S)$. These edges are called Voronoi edges and the vertices are called Voronoi vertices. A region $\operatorname{reg}(p)$ is called a Voronoi cell (or Voronoi polygon).

\section{Proposed Method}

In the proposed approach, in general the conventional Delaunay Triangulation depending on the ball position is used as the method for positioning but in the attacking and near opponent's penalty area and cross situations, due to increasing development of marking abilities and defense power of 2D SS teams, scoring goal has become a hard and challenging task. Attackers should escape the marking of the opponent's defenders in order to create space for them to have the chance to score.

For this we apply the "Voronoi Diagram" idea and "Maximum Weighted Bipartite Matching". The algorithm is as follows. First the Voronoi diagram of opponent's defenders positions is created and the vertices of the diagram are found. Considering factors such as offside line and the fact that the vertex lies on the field (not out of field or near out of field) some vertices from the possible choices are omitted. The remaining vertices of the opponent's agents Voronoi Diagram are considered as potential positioning points for attacking team agents, since the vertices of the Voronoi diagram are good positions for the attackers because they have safe distance from opponent's defenders and positioning in

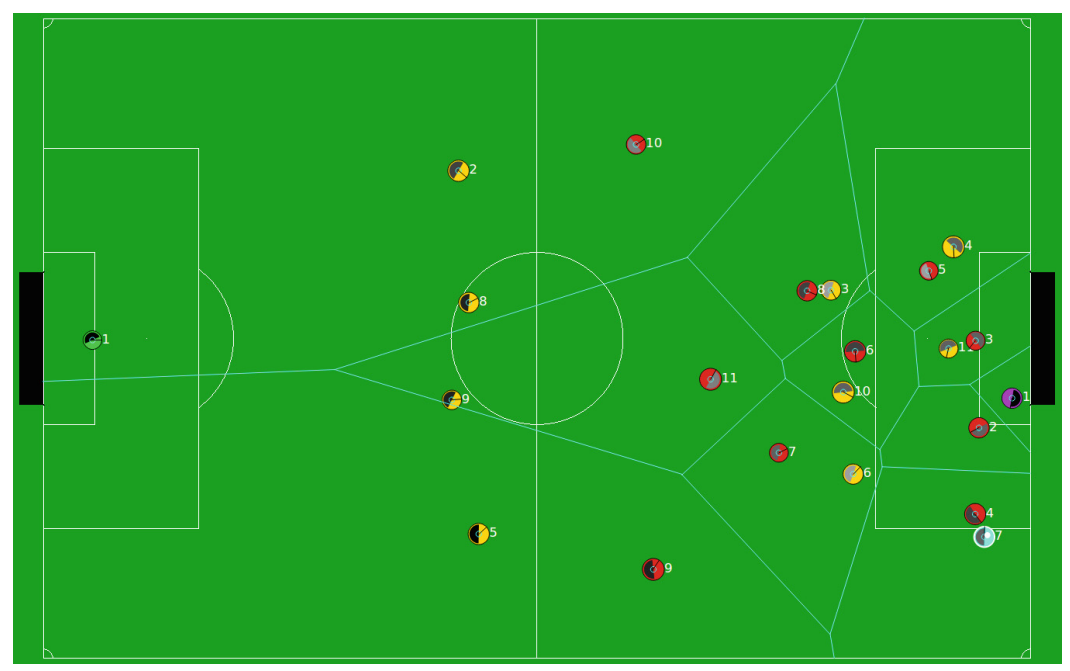

Fig. 2. Potential positioning places based on Voronoi diagram of the defending team's agents (red agents) (Color figure online). 
these points increases the chance of receiving passes from teammate agent ball owner, as can be seen in Fig. 2. In Fig. 2 the Voronoi Diagram of the defending team agents (red agents) is depicted, and it can be seen that the vertices of this diagram are potential safe and good positions for the attacking team agents (yellow agents) to take position on. For assigning the attackers to vertices positions without any conflict we apply "MWBM" to our problem employing Hungarian algorithm for solving it. The attackers are assumed on one side and the vertices positions on the other side as the graph's nodes. Then a weight $w(i, j)$ is given to each edge respecting some features. The weight in fact indicates the importance and priority of choosing the point $j$ by the attacker $i$ for positioning.

In fact, if $F_{k}$ is the value of feature $k$ and $R_{k}$ is the related coefficient and $N$ is the number of the features the weight of each edge is the sum of the features value multiplied by their related coefficients as shown in (6).

$$
W_{(i, j)}=\sum_{k=1}^{N} R_{k} \times F_{k}(i, j)
$$

Some of the factors that should be taken into account are the ones that have a great effect on stamina consumption of the agents. Factors like distance from current position or distance from formation position. In fact by considering these factors another goal is achieved in which the least deviation from the pre determined formation positions exists.

Some of the important elements in the weight calculation are shown in the Table 1.

In order to prevent the possible conflicts in applying the algorithm by each agent individually, e.g. choosing the same vertex for positioning by two or more agents due to different final matches concluded by each agent individually which is the result of the limitations of information of the current field situation, one of the agents who have the best view in attacking situations executes the matching and informs the attacker agents of the result by using "say ability".

\section{Tests and Results}

For comparing our algorithm in attacking situations with the conventional Delaunay Triangulation depending just on ball position, we employ the agent 2D 3.1.1 source code (team) which is released by Helios Soccer Simulation Team and is the base code of most of the participant teams in 2D Soccer Simulation League of Robocup. We have conducted two tests: one with its conventional and default method of positioning and the other with our modification in attacking situation. In both cases the formation file and settings are the same and the default version of agent $2 \mathrm{~d}$ 3.1.1 formation designed in fedit software is used. We tested both scenarios against the same binary files of famous teams of around the world participating in World Robocup Competitions. In these tests parameters such as 
Table 1. Important factors of our positioning decision

\begin{tabular}{|c|c|c|c|}
\hline Factor & Feature & Description & Value \\
\hline $\mathrm{F} 1$ & $\begin{array}{l}\text { Opponent mean } \\
\text { distance }\end{array}$ & $\begin{array}{l}\text { The opponent's agents } \\
\text { mean distance to the } \\
\text { position }\end{array}$ & $\begin{array}{l}\text { The higher the distance } \\
\text { is, the higher value is } \\
\text { allocated to } \mathrm{F} 1\end{array}$ \\
\hline F2 & $\begin{array}{l}\text { Home position } \\
\text { distance }\end{array}$ & $\begin{array}{l}\text { The distance between the } \\
\text { formation position of the } \\
\text { attacker and the vertex } \\
\text { position }\end{array}$ & $\begin{array}{l}\text { The lower the distance } \\
\text { is, the higher value is } \\
\text { allocated to } \mathrm{F} 2\end{array}$ \\
\hline F3 & Formation changing & $\begin{array}{l}\text { The amount of the mess of } \\
\text { our formation with that } \\
\text { positioning }\end{array}$ & $\begin{array}{c}\text { The less the amount is, } \\
\text { the higher value is } \\
\text { allocated to F3 }\end{array}$ \\
\hline F4 & Distance from goal & $\begin{array}{l}\text { The distance between the } \\
\text { vertex and opponent's } \\
\text { goal }\end{array}$ & $\begin{array}{l}\text { The lower the distance } \\
\text { is, the higher value is } \\
\text { allocated to F4 }\end{array}$ \\
\hline F5 & $\begin{array}{l}\text { Current position } \\
\text { distance }\end{array}$ & $\begin{array}{l}\text { The distance between the } \\
\text { vertex and the current } \\
\text { position of attacker }\end{array}$ & $\begin{array}{l}\text { The lower the distance } \\
\text { is, the higher value is } \\
\text { allocated to F5 }\end{array}$ \\
\hline F6 & Distance to ball & $\begin{array}{l}\text { The distance between the } \\
\text { position and ball } \\
\text { position }\end{array}$ & $\begin{array}{l}\text { In a specified range the } \\
\text { longer the distance } \\
\text { is, the higher value is } \\
\text { allocated to F6; and } \\
\text { in other cases the } \\
\text { shorter the distance } \\
\text { is, the higher value is } \\
\text { allocated }\end{array}$ \\
\hline F7 & $\begin{array}{l}\text { Distance to } \\
\text { teammate }\end{array}$ & $\begin{array}{l}\text { The position's distance } \\
\text { from our nearest } \\
\text { teammate to that } \\
\text { position }\end{array}$ & $\begin{array}{l}\text { In a specified range the } \\
\text { longer the distance } \\
\text { is, the higher value is } \\
\text { allocated to F7; and } \\
\text { in other cases the } \\
\text { shorter the distance } \\
\text { is, the higher value is } \\
\text { allocated }\end{array}$ \\
\hline
\end{tabular}

the number of completed passes in opponent's half-field, number of goals scored and number of cycles ball was owned that are suitable features for this comparison were measured. Each match was run three times. The measured mean values are shown in Table 2. The resultant values in tests against a small selection of teams participated in 2013 Robocup competitions are also shown in Table 3 . In addition, the values measured against the agent 2D 3.1.1 base code itself is also reported in Table 3. These tables show that the number of correct passes and goals scored are increased by employing the proposed method. The number of cycles which the team owns the ball, however, have been decreased.Although it might seem unacceptable, due to rather more stamina consumption caused 
Table 2. Total measured values and test results

\begin{tabular}{l|l|l|l}
\hline Factor & Measure & Description & $\begin{array}{l}\text { Value(D:Delaunay/ } \\
\text { V:Voronoi) }\end{array}$ \\
\hline M1 & Successful pass & $\begin{array}{l}\text { The average number of } \\
\text { complete passes in the } \\
\text { opponent half-field in } \\
\text { each match }\end{array}$ & D: 26.33, V: 38.46 \\
\hline M2 & Goals scored & $\begin{array}{l}\text { The average number of } \\
\text { goals scored in each } \\
\text { match } \\
\text { The average number of } \\
\text { cycles the team owns the } \\
\text { ball in each match }\end{array}$ & D: $1.33, \mathrm{~V}: 1.56$ \\
\hline M3 & Possession cycles & \multicolumn{2}{|c|}{$2184.5, \mathrm{~V}: 1995.7$} \\
\hline
\end{tabular}

Table 3. Test results against a selection of teams

\begin{tabular}{l|l|l|l}
\hline Rival team & $\begin{array}{l}\text { Successful pass } \\
\text { (D:Delaunay/ } \\
\text { V:Voronoi) }\end{array}$ & $\begin{array}{l}\text { Goals scored } \\
\text { (D:Delaunay/ } \\
\text { V:Voronoi) }\end{array}$ & $\begin{array}{l}\text { Possession cycles } \\
\text { (D:Delaunay/ } \\
\text { V:Voronoi) }\end{array}$ \\
\hline AUT & D: $41, \mathrm{~V}: 63.66$ & D: 1.33 V: 1.66 & D: 3180.33, V: 2850.33 \\
\hline Helios & D: 22.33, V: 31.33 & D: $0.0, V: 0.0$ & D: $1650, \mathrm{~V}: 1400.33$ \\
\hline Yushan & D: $31.66, V: 37.33$ & D: $0.0, V: 0.33$ & D: 2440.33, V: 2750.33 \\
\hline agent 2D & D: $22, V: 26.33$ & D: $1.66, V: 4.33$ & D: $2790.66, V: 2410$ \\
\hline
\end{tabular}

by the method's sensitiveness to the opponent agents, this occurrence is obvious. This algorithm has also been used in our AUT Soccer Simulation team since 2012 and the great number of goals scored by this team in different competitions such as Robocup 2012, Iranopen 2013 and Robocup 2013 is another indicator of this algorithm acceptable performance. The coefficients $R_{k}$ mentioned in Sect. 4 that are used for scoring method regarding the features can be determined by the developer as a human observer and expert or can be optimized by defining optimization scenarios and using advanced AI methods to improve the performance of the method.

\section{Conclusion}

We proposed a method for attacking situation positioning of the agents in $2 \mathrm{D}$ Soccer Simulation, but its application is not restricted to the 2D SS League. The proposed method employs the vertices of the Voronoi Diagram of the defending team agents as potential positions for the attacker team agents. It then applies Maximum Weighted Bipartite Matching to match these vertices to the attacking agents. As shown in the previous section this method shows better performance 
in offensive situation than the conventional Delaunay Triangulation based positioning. But as mentioned in Sect. 5, this method is more sensitive to opponent agents movements, and might consume more stamina by causing some redundant move actions of the agents. Another fact is that the defensive skills of soccer teams are more effective near their goal, and escaping from the advanced marking skills of opponent team agents is very important in cross situations and near goal situations in order to score more goals. Therefore, our opinion is that the proposed method should be used mostly in cross situations and near goal and penalty area situations, and for the attacking moments that the ball is far from the defending team penalty area a simple free run behavior can be performed by the attacking agents in order to save more stamina and increase the chance of receiving through passes by them. This is the same combined method employed in our AUT Soccer Simulation team, which has shown good performance in different Robocup competitions.

\section{References}

1. Dashti, H.T., Kamali, S., Aghaeepour, N.: Positioning in robots soccer. In: Lima, P. (ed.) Robotic Soccer, pp. 29-44. I-Tech Education and Publishing, Austria (2007)

2. Kaden, S., Mellmann, H., Scheunemann, M., Burkhard, H.D.: Voronoi based strategic positioning for robot soccer. In: Proceedings of the 22nd International Workshop on Concurrency, Specification, and Programming (CS\&P), Warsaw, pp. 283$293(2013)$

3. Akiyama, H., Noda, I.: Multi-agent positioning mechanism in the dynamic environment. In: Visser, U., Ribeiro, F., Ohashi, T., Dellaert, F. (eds.) RoboCup 2007: Robot Soccer World Cup XI. LNCS (LNAI), vol. 5001, pp. 377-384. Springer, Heidelberg (2008)

4. Akiyama, H., Noda, I., Shimora, H.: Helios 2008 team description paper. In: RoboCup (2008)

5. Akiyama, H.: Helios 2007 team description paper. In: RoboCup (2007)

6. Part of Helios team released materials. http://sourceforge.jp/projects/rctools/

7. Lecture notes from Michael Goemans class on Combinatorial Optimization. http:// math.mit.edu/ goemans/18433S09/matching-notes.pdf (2009)

8. Paul, M.: Algorithmen fur das maximum weight matching problem in bipartiten graphen. Master's thesis, Fachbereich Informatik, Universität des Saarlandes, Saarbrücken (1989)

9. Kreyszig, E.: Advanced Engineering Mathematics, 10th edn. Wiley, Hoboken (2010)

10. West, D.B.: Introduction to Graph Theory, 2nd edn. Prentice Hall, Englewood Cliffs (1999)

11. Norouzitallab, M., Javari, A., Noroozi, A., Salehizadeh, S.M.A., Meshgi, K.: Nemesis team description paper. In: RoboCup (2010)

12. Malmir, M., Simchi, M., Boluki, S.: AUT Team Description Paper 2012. In: RoboCup (2012)

13. Aurenhammer, F.: Voronoi diagrams - a survey of a fundamental geometric data structure. ACM Comput. Surv. 23(4), 345-406 (1991)

14. Berg, M., Cheong, O., Kreveld, M., Overmars, M.: Computational Geometry: Algorithms and Applications. Springer, Berlin (2008)

15. Wang, B.: Coverage Control in Sensor Networks. Springer, New York (2010) 